\title{
Spectral response of fixed photorefractive grating interference filters
}

\author{
Rudolf Hofmeister, Amnon Yariv, and Shogo Yagi* \\ California Institute of Technology, Pasadena, California 91125
}

\begin{abstract}
Received October 5, 1992; revised manuscript received October 28, 1993; accepted October 28, 1993
We report a theoretical investigation of the frequency response of optical interference filters written in photorefractive materials. Counterpropagating coherent beams interact in the volume of a photorefractive crystal through two-beam coupling. The resulting hologram is fixed. The reflectivity of the hologram is calculated as a function of frequency. An analytic solution is obtained for arbitrary grating phase $\phi$ in the lossless case, $\alpha=0$. Numerical solutions are performed for $\alpha>0$. Experimental results are compared favorably with the theory.
\end{abstract}

\section{INTRODUCTION}

Fixed photorefractive gratings have received interest recently for application as narrow-band optical interference filters and wavelength multiplexed optical memories. ${ }^{1-4}$ The advantages of photorefractive filters over conventional methods are ease of fabrication and extremely narrow spectral response. We consider Bragg gratings that are written in a photorefractive material in exactly counterpropagating geometry. This method allows one to tune the bandpass of the filter by adjusting the frequency of the laser writing beams. The index gratings written by the beams are then considered to be fixed by either thermal fixing or other methods. We calculate the spectral response of these filters to broadband or tunable incident radiation. These results are also valid for dynamic gratings if the gratings are illuminated for a period of time that is much shorter than the grating rewrite time.

\section{SPECTRAL RESPONSE, LOSSLESS CASE}

Calculation of the Dynamic Index Grating

We start by calculating the two-beam coupling of two incident counterpropagating beams $A(z)$ and $B(z)$ in a photorefractive material. The well-known coupled-mode equations in the absence of optical absorption are ${ }^{5}$

$$
\begin{aligned}
& A^{\prime}(z)=\frac{i \pi \Delta n(z)}{\lambda} e^{i \phi} B(z), \\
& B^{\prime}(z)=\frac{-i \pi \Delta n^{*}(z)}{\lambda} e^{-i \phi} A(z),
\end{aligned}
$$

where $\lambda$ is the wavelength of the interfering beams and the index of refraction is

$$
n(z)=n_{0}+1 / 2\left[\Delta n(z) e^{i \phi} \exp (i 2 k z)+\text { c.c. }\right] \text {. }
$$

Here $\phi$ is the phase between the optical intensity grating and the induced index grating. This grating phase is an invariant property of the material; it can be altered with applied electric fields but is independent of the illuminating beams. If the photorefractive response in the material is given purely by the diffusion field, i.e., there is no photovoltaic effect or applied electric field, then the phase obeys $\phi=\pi / 2{ }^{6,7}$ We require, without loss of generality, that $B(z)$ be the amplified beam. That is, the propagation direction of $B(z)$ is nearer the $z$ axis ( $c$ axis) is than that of $A(z)$. In exactly counterpropagating geometry as in Eqs. (1) and (2), $B(z)$ propagates parallel to the optic axis and $A(z)$ propagates antiparallel to the same. This is equivalent to restricting $\phi$ to the range $[0, \pi]$. Since the index grating is formed dynamically by the writing beams, we have

$$
\Delta n(z)=n_{1} A(z) B^{*}(z) / I(z),
$$

where $I(z)$ is the total intensity, $I(z)=\left|A^{2}(z)\right|+\left|B^{2}(z)\right|$, and $n_{1}$ is the peak-to-peak amplitude of the index grating when $A(z)=B(z)$. Thus in the case of dynamic holography the coupled-mode equations are rewritten as

$$
\begin{aligned}
& A^{\prime}(z)=i g|B(z)|^{2} / I(z) e^{i \phi} A(z), \\
& B^{\prime}(z)=-i g|A(z)|^{2} / I(z) e^{-i \phi} B(z),
\end{aligned}
$$

with $g=\pi n_{1} / \lambda$. We postulate solutions of the form

$$
A(z)=a(z) e^{i \psi_{1}}, \quad B(z)=b(z) e^{i \psi_{2}},
$$

where $a(z)$ and $b(z)$ are real. Equations (4a) and (4b) can be separated into two equations each, describing the evolution of the amplitude and the phase, respectively, of the two beams:

$$
\begin{aligned}
a^{\prime}(z) & =-g \sin (\phi) b(z)^{2} / I(z) a(z), \\
b^{\prime}(z) & =-g \sin (\phi) a(z)^{2} / I(z) b(z), \\
\psi_{1}{ }^{\prime}(z) & =g \cos (\phi) b(z)^{2} / I(z), \\
\psi_{2}{ }^{\prime}(z) & =-g \cos (\phi) a(z)^{2} / I(z) .
\end{aligned}
$$

Inspection of Eqs. (6) reveals that the intensity coupling, commonly referred to as beam coupling, increases from 


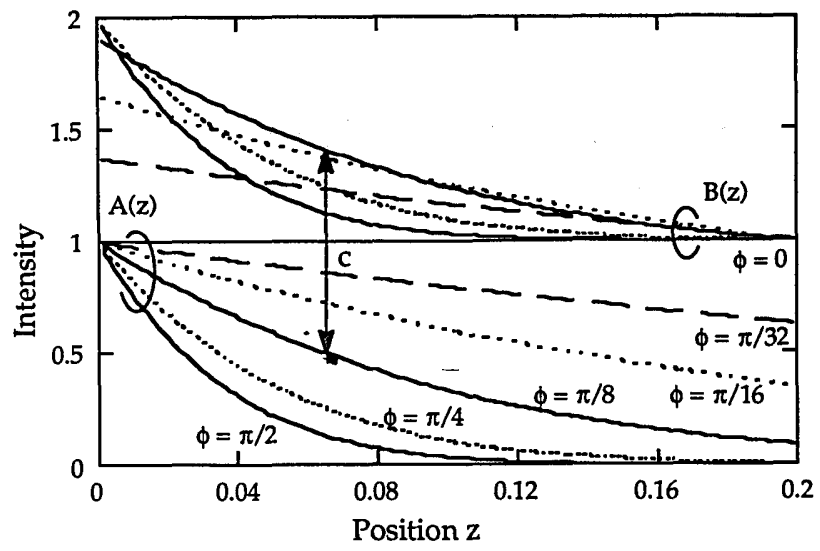

Fig. 1. Effect of the photorefractive grating phase $\phi$ on the intensity coupling of two equal-intensity input beams. For $\phi=0$ the intensity coupling is zero and the beams are purely phase coupled. As $\phi$ deviates from zero, the beams are coupled more and more strongly near the entrance face of the crystal. The coupling constant is $g=20 / \mathrm{cm}$, and the crystal length is $L=0.2 \mathrm{~cm}$.

zero to a maximum value as the photorefractive phase deviates from 0 to $\pi / 2$. Meantime, Eqs. (7) show that the phase coupling of the two beams decreases from a maximum, when $\phi=0$, to zero, when $\phi=\pi / 2$. Thus in the diffusion-limited case mentioned above $(\phi=\pi / 2)$, the beam coupling in the material achieves its maximum value, while the phase coupling is zero.

One solves Eqs. (6a) and (6b) by converting them to equations for intensities, using $I_{1}{ }^{\prime}=\left(a^{2}\right)^{\prime}=2 a a^{\prime}$, and similarly for $I_{2}$, respectively. Simple Bernoulli equations are obtained with solutions ${ }^{8,9}$

$$
\begin{aligned}
& I_{1}(z)=1 / 2\left[\left(c^{2}+v^{2} e^{-\Gamma z}\right)^{1 / 2}+c\right], \\
& I_{2}(z)=1 / 2\left[\left(c^{2}+v^{2} e^{-\Gamma z}\right)^{1 / 2}-c\right] .
\end{aligned}
$$

Here we have used the constants $\Gamma=2 \mathrm{~g} \sin \phi$, $c=I_{1}(z)-I_{2}(z)$, and $v^{2}=4 I_{1}(0) I_{2}(0)=4 I_{1}(z) I_{2}(z) e^{\Gamma z}=$ $4 I_{1}(0)\left[I_{1}(0)-c\right]$. Also, $I_{1}(z)=|A(z)|^{2}$ and $I_{2}(z)=|B(z)|^{2}$. In terms of the input intensities to the crystals, $I_{1}(0)$ and $I_{2}(L)$, the constants are given by

$$
\begin{aligned}
c & =\frac{e^{-\Gamma L} I_{1}^{2}(0)-I_{2}^{2}(L)}{e^{-\Gamma L} I_{1}(0)+I_{2}(L)}, \\
v^{2} & =4 I_{1}(0) I_{2}(L) \frac{I_{1}(0)+I_{2}(L)}{e^{-\Gamma L} I_{1}(0)+I_{2}(L)}
\end{aligned}
$$

In the case of equal-intensity inputs $\left[I_{1}(0)=I_{2}(L)\right]$, these constants reduce to $v^{2}=4 I_{1}^{2}(0) \exp (\Gamma L / 2) / \cosh (\Gamma L / 2)$ and $c=-I_{1}(0) \tanh (\Gamma L / 2)$. We note that $c$ as it is defined is a constant by conservation of energy. This follows immediately from the coupled equations, whereby any decrease in the intensity of one beam is added to the intensity of the other beam, which is propagating in the opposite direction. ${ }^{5,8}$ The intensities are plotted in Fig. 1 for the case of equal-intensity inputs, for coupling constant $g=20 / \mathrm{cm}$, crystal length $L=0.2 \mathrm{~cm}$, and several values of $\phi$. The intensity coupling increases as the photorefractive phase $\phi$ deviates from zero.

From the solutions given in Eq. (8) we readily solve Eqs. 7(a) and 7(b) to give

$$
\psi_{1}(z)=1 / 2\left(g \cos (\phi) z-\cot (\phi) \operatorname{coth}^{-1}\left\{\left[1+(v / c)^{2} e^{-\Gamma z}\right]^{1 / 2}\right\}\right),
$$

$$
\begin{aligned}
\psi_{2}(z)= & -1 / 2\left(g \cos (\phi) z+\cot (\phi) \operatorname{coth}^{-1}\right. \\
& \left.\times\left\{\left[1+(v / c)^{2} e^{-\Gamma z}\right]^{1 / 2}\right\}\right)
\end{aligned}
$$

respectively. Thus from Eqs. (5), (8), and (10) we have solved the beam coupling in the counterpropagating geometry for arbitrary input beams and arbitrary material grating phase $\phi$. The index grating in the material follows from Eqs. (2) and (3) and is given by

$$
\Delta n e^{i \phi}=\frac{n_{1}}{2} \frac{e^{+i \phi} \exp [+i g \cos (\phi) z]}{\left(1+c^{2} e^{\Gamma z} / v^{2}\right)^{1 / 2}} .
$$

The functional form of the index grating is simplified by the partial cancellation of the beam phases in Eqs. (10). The spatial variation of the magnitude of the index grating is plotted in Fig. 2 for the case of equal-intensity inputs and for several values of $\phi$. It is illuminating to note that the maximum value of the index grating amplitude always occurs at the entrance facet of the crystal in the direction of the $c$ axis, i.e., in the plane $z=0$. (Experimentally, one can distinguish the plane $z=0$ from the plane $z=L$ by inputting two equal-intensity beams into the crystal in the counterpropagating geometry. The amplified beam is the one that exits from the $z=0$ facet.) This is true regardless of the incident-beam intensity ratios. This peculiar result originates in the nature of the intensity coupling shown in Fig. 1: both the incident and the reflected beam achieve their maximum intensities at the $z=0$ entrance facet, and the difference between the two beam intensities is always a constant $(=c)$. The combination of these two facts ensures, by Eq. (3), that the index grating amplitude is maximum at $z=0$.

\section{Calculation of Reflectivity}

We calculate the frequency reflectivity of an incident beam $A(0)$ off the dynamically written index grating. In

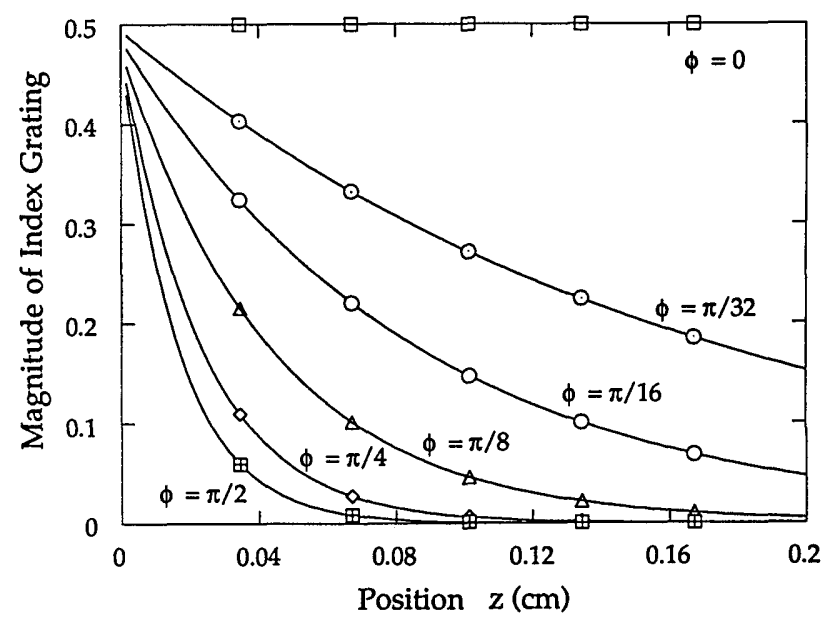

Fig. 2. Magnitude of the index grating formed by the intensity coupled beams of Fig. 1. For $\phi=0$ the index grating is constant, and as $\phi$ increases, the index grating is more strongly apodized, with its maximum at the entrance face of the crystal. 
analogy to Eqs. (1a) and (1b), we write the coupled-mode equations, ${ }^{10}$

$$
\begin{aligned}
& A^{\prime}(z)=i \frac{g}{2} \frac{\exp [i g \cos (\phi) z+i \phi]}{\left(1+c^{2} e^{\Gamma z} / v^{2}\right)^{1 / 2}} \exp (-2 i \Delta \beta z) B(z), \\
& B^{\prime}(z)=-i \frac{g}{2} \frac{\exp [-i g \cos (\phi) z-i \phi]}{\left(1+c^{2} e^{\Gamma z} / v^{2}\right)^{1 / 2}} \exp (+2 i \Delta \beta z) A(z) \text {, }
\end{aligned}
$$

respectively, where $\Delta \beta=\left(\omega_{0}-\omega\right) n_{0} / c$ is the frequency mismatch between the beams that wrote the grating and the one undergoing Bragg reflection. We have ignored the new dynamic grating that is written by the interference of the incident and the reflected beams, ${ }^{11,12}$ since we are calculating the filter response to broadband illumination. Any such secondary grating would be bleached by the majority of the light that is not reflected. In Eqs. (12), $B(z)$ is the new reflected beam, so that we take $B(L)=0$, where $L$ is the length of the crystal. The analytic solution of Eqs. (12) subject to this boundary condition follows. The interested reader will observe similarities in the approach presented here to a recent treatment of diffraction in the transmission geometry. ${ }^{13}$

We first note that Eqs. (12a) and (12b) can be written in the form

$$
\begin{aligned}
& A^{\prime}(z)=i g(z) f(z) B(z), \\
& B^{\prime}(z)=-i[g(z) / f(z)] A(z),
\end{aligned}
$$

respectively, where $f(z)=\exp [-2 i \Delta \beta z+i g \cos (\phi) z+$ $i \phi]$ and $g(z)=g v \exp (-\Gamma z / 2) /\left\{2\left[c^{2}+v^{2} \exp (-\Gamma z)\right]^{1 / 2}\right\}$. We can eliminate $g(z)$ by performing the independent variable transformation of $z$ to $\xi$, where $\xi / \sin \phi=$ $\int g(z) \mathrm{d} z$ :

$$
\begin{aligned}
\xi & =-\frac{\Gamma v}{2 c} \int \frac{\exp (-\Gamma z / 2)}{\left[1+(v / c)^{2} \exp (-\Gamma z)\right]^{1 / 2}} \mathrm{~d} z \\
& =\sinh ^{-1}\left[\frac{v}{c} \exp (-\Gamma z / 2)\right] .
\end{aligned}
$$

Inverting, we obtain $\exp (-\Gamma z / 2)=(c / v) \sinh (\xi)$. Applying this transform to Eqs. (12a) and (12b) yields a set of coupled equations in $\xi$,

$$
\begin{aligned}
& a^{\prime}(\xi)=i \kappa f(\xi) b(\xi), \\
& b^{\prime}(\xi)=-i \kappa^{*} f^{*}(\xi) a(\xi),
\end{aligned}
$$

respectively, where

$$
\begin{aligned}
f(\xi) & =\left[\frac{c}{v} \sinh (\xi)\right]^{4 i \Delta \beta^{\prime} / \Gamma}, \\
\kappa & =-e^{i \phi} /(2 \sin \phi),
\end{aligned}
$$

and $\Delta \beta^{\prime}=\Delta \beta-(g / 2) \cos \phi$. In Eqs. (14), the lowercase of $a$ and $b$ is used only to remind the reader of the change in independent variable; thus $A(z), B(z) \rightarrow a(\xi), b(\xi)$.

Reference to Appendix A reveals that Eqs. (14a) and (14b) are of the form of Eqs. (A1a) and (A1b), respectively, with $\delta=0$, and can be transformed with the corotating transform developed there. We define

$$
a(\xi)=T(\xi) \exp [i F(\xi)], \quad b(\xi)=V(\xi) \exp [-i F(\xi)]
$$

where

$$
F(\xi)=1 /(2 i) \ln [f(\xi)]=\Delta \beta^{\prime} /(g \sin \phi) \ln [\sinh (\xi)]
$$

Second-order equations in $T(\xi), T^{\prime \prime}(\xi)$ and $V(\xi), V^{\prime \prime}(\xi)$ are obtained as they are given by Eqs. (A8). As is described in Appendix A, we chose $F(\xi)$ to eliminate the first derivative terms $T^{\prime}(\xi)$ and $V^{\prime}(\xi)$ in Eqs. (A8). From Eq. (18) we can write $F^{\prime}(\xi)=\Delta \beta^{\prime} /(g \sin \phi) \operatorname{coth}(\xi)$ and $F^{\prime \prime}(\xi)=$ $-\Delta \beta^{\prime} /\left[g \sin \phi \sinh ^{2}(\xi)\right]$ so that Eqs. (A8) become

$$
\begin{gathered}
T^{\prime \prime}(\xi)+\left[\frac{-1}{4 \sin ^{2} \phi}+\eta^{2}-\frac{i \eta(i \eta+1)}{\sinh ^{2} \xi}\right] T(\xi)=0, \\
V^{\prime \prime}(\xi)+\left[\frac{-1}{4 \sin ^{2} \phi}+\eta^{2}-\frac{i \eta(i \eta-1)}{\sinh ^{2} \xi}\right] V(\xi)=0,
\end{gathered}
$$

where we define $\eta=2 \Delta \beta^{\prime} / \Gamma$. Comparison with Eq. (A9) confirms that Eqs. (19a) and (19b) are examples of the second Pöschl-Teller equation, where $\lambda=0, \alpha=1$, and the quantum parameters are

$$
\begin{aligned}
l & =\frac{1}{2}\left(\frac{1}{4 \sin ^{2} \phi}-\eta^{2}\right)^{1 / 2}+\frac{1}{2}, \\
\kappa_{T} & =1+i \eta, \\
\kappa_{v} & =i \eta .
\end{aligned}
$$

The parameters $\kappa_{T}$ and $\kappa_{V}$ are those required for Eqs. (19a) and (19b), respectively, to match the notation of Eq. (A9). The quantity $l$ is the angular-momentum quantum number in the diatomic system. Here it assumes not only noninteger values but, for $\Delta \beta^{\prime}>$ $g \sin \phi / 2$, becomes complex. Thus little intuition can be drawn from the quantum system to help us in this application. Fortunately, the solution of the Pöschl-Teller equation works with complex parameters and can be utilized as is.

Using Refs. 14 and 15, we obtain solutions for $T(\xi)$ and $V(\xi)$ :

$$
\begin{aligned}
T(\xi)= & t_{1} \sinh ^{-i \eta} \xi \cosh (\xi)_{2} F_{1}\left(\frac{1}{2}-i \frac{\eta}{2}-\beta, \frac{1}{2}-i \frac{\eta}{2}\right. \\
& \left.+\beta ; \frac{1}{2}-i \eta ;-\sinh ^{2} \xi\right) \\
& +t_{2} \sinh ^{1+i \eta} \xi \cosh (\xi)_{2} F_{1}\left(i \frac{\eta}{2}-\beta+1, i \frac{\eta}{2}\right. \\
& \left.+\beta+1 ; \frac{3}{2}+i \eta ;-\sinh ^{2} \xi\right), \\
V(\xi)= & v_{1} \sinh ^{1-i \eta} \xi \cosh (\xi){ }_{2} F_{1}\left(1-i \frac{\eta}{2}-\beta, 1-i \frac{\eta}{2}\right. \\
& \left.+\beta ; \frac{3}{2}-i \eta ;-\sinh ^{2} \xi\right) \\
& +v_{2} \sinh ^{i \eta} \xi \cosh (\xi){ }_{2} F_{1}\left(i \frac{\eta}{2}-\beta+\frac{1}{2}, i \frac{\eta}{2}\right. \\
& \left.+\beta+\frac{1}{2} ; \frac{1}{2}+i \eta ;-\sinh ^{2} \xi\right),
\end{aligned}
$$


where $t_{1}, t_{2}, v_{1}$, and $v_{2}$ are arbitrary constants, and we define

$$
\beta=\frac{1}{2}\left(\frac{1}{4 \sin ^{2} \phi}-\eta^{2}\right)^{1 / 2}=l-\frac{1}{2} .
$$

$\beta$ is distinct from the frequency mismatch $\Delta \beta$. Using Eqs. (13), (14), and (17), we get

$$
\begin{aligned}
A(z)= & {\left[1+\left(\frac{v}{c}\right)^{2} e^{-\Gamma z}\right]^{1 / 2}\left\{C _ { 1 2 } F _ { 1 } \left[\frac{1}{2}-i \frac{\eta}{2}-\beta, \frac{1}{2}\right.\right.} \\
& \left.-i \frac{\eta}{2}+\beta ; \frac{1}{2}-i \eta ;-\left(\frac{v}{c}\right)^{2} e^{-\Gamma z}\right] \\
& +C_{2} \exp [-(1+2 i \eta) \Gamma z / 2]_{2} F_{1}\left[i \frac{\eta}{2}-\beta\right. \\
+ & \left.\left.1, i \frac{\eta}{2}+\beta+1 ; \frac{3}{2}+i \eta ;-\left(\frac{v}{c}\right)^{2} e^{-\Gamma z}\right]\right\}, \quad(25 \mathrm{a}) \\
B(z)= & {\left[1+\left(\frac{v}{c}\right)^{2} e^{-\Gamma z}\right]^{1 / 2}\left\{C_{3} \exp [-(1-2 i \eta) \Gamma z / 2]\right.} \\
& \times{ }_{2} F_{1}\left[-i \frac{\eta}{2}-\beta+1,-i \frac{\eta}{2}+\beta+1 ; \frac{3}{2}\right. \\
& \left.-i \eta ;-\left(\frac{v}{c}\right)^{2} e^{-\Gamma z}\right]+C_{42} F_{1}\left[i \frac{\eta}{2}-\beta\right. \\
& \left.\left.+\frac{1}{2}, i \frac{\eta}{2}+\beta+\frac{1}{2} ; \frac{1}{2}+i \eta ;-\left(\frac{v}{c}\right)^{2} e^{-\Gamma z}\right]\right\}
\end{aligned}
$$

where $C_{1}, C_{2}, C_{3}$, and $C_{4}$ are constants. We determine the constants from the boundary condition $B(L)=0$ and Eqs. (12). The result is

$$
\begin{aligned}
C_{1}= & \frac{A(0) 4 \sin ^{2}(\phi)\left(1+4 \eta^{2}\right)}{\left[1+(v / c)^{2}\right]^{1 / 2}} \exp [(1-2 i \eta) \Gamma L / 2] \\
& \times{ }_{2} F_{1}\left[i \frac{\eta}{2}-\beta+\frac{1}{2}, i \frac{\eta}{2}+\beta\right. \\
& \left.+\frac{1}{2} ; \frac{1}{2}+i \eta ;-\left(\frac{v}{c}\right)^{2} e^{-\Gamma L}\right] / D \\
C_{2}= & \frac{-A(0)}{\left[1+(v / c)^{2}\right]^{1 / 2}}\left(\frac{v}{c}\right)^{2}{ }_{2} F_{1}\left[-i \frac{\eta}{2}-\beta+1,-i \frac{\eta}{2}\right. \\
& \left.+\beta+1 ; \frac{3}{2}-i \eta ;-\left(\frac{v}{c}\right)^{2} e^{-\Gamma L}\right] / D, \\
C_{3}= & -A(0)\left\{\frac{v(2+4 i \eta) \sin \phi}{i c e^{i \phi}\left[1+(v / c)^{2}\right]^{1 / 2}}\right\} \exp [(1-2 i \eta) \Gamma L / 2] \\
& \times{ }_{2} F_{1}\left[i \frac{\eta}{2}-\beta+\frac{1}{2}, i \frac{\eta}{2}+\beta\right. \\
& \left.+\frac{1}{2} ; \frac{1}{2}+i \eta ;-\left(\frac{v}{c}\right)^{2} e^{-\Gamma L}\right] / D, \\
C_{4}= & A(0)\left\{\frac{v(2+4 i \eta) \sin \phi}{i c e^{i \phi}\left[1+(v / c)^{2}\right]^{1 / 2}}\right\}_{2} F_{1}\left[-i \frac{\eta}{2}-\beta\right. \\
& \left.+1,-i \frac{\eta}{2}+\beta+1 ; \frac{3}{2}-i \eta ;-\left(\frac{v}{c}\right)^{2} e^{-\Gamma L}\right] / D,
\end{aligned}
$$

where the common denominator $D$ is given by

$$
\begin{aligned}
D= & 4 \sin ^{2}(\phi)\left(1+4 \eta^{2}\right) \exp [(1-2 i \eta) \Gamma L / 2] \\
& \times{ }_{2} F_{1}\left[i \frac{\eta}{2}-\beta+\frac{1}{2}, i \frac{\eta}{2}+\beta+\frac{1}{2} ; \frac{1}{2}\right. \\
& \left.+i \eta ;-\left(\frac{v}{c}\right)^{2} e^{-\Gamma L}\right]{ }_{2} F_{1}\left[-i \frac{\eta}{2}-\beta\right. \\
& \left.+\frac{1}{2},-i \frac{\eta}{2}+\beta+\frac{1}{2} ; \frac{1}{2}-i \eta ;-\left(\frac{v}{c}\right)^{2}\right] \\
& -\left(\frac{v}{c}\right)_{2}^{2} F_{1}\left[-i \frac{\eta}{2}-\beta+1,-i \frac{\eta}{2}+\beta\right. \\
& \left.+1 ; \frac{3}{2}-i \eta ;-\left(\frac{v}{c}\right)^{2} e^{-\Gamma L}\right] \\
& \times{ }_{2} F_{1}\left[i \frac{\eta}{2}-\beta+1, i \frac{\eta}{2}\right. \\
& \left.+\beta+1 ; \frac{3}{2}+i \eta ;-\left(\frac{v}{c}\right)^{2}\right] .
\end{aligned}
$$

Equations (25) with constant coefficients defined in Eqs. (26) and (27) determine exactly the amplitude and phase of the incident beam $A(z)$ and the reflected beam $B(z)$.

To recap, the reflected wave is generated by interaction of $A(z)$ with the previously dynamically written hologram defined by the index grating of Eq. (11). Since $B(z)$ is a reflected wave, the solutions obey the boundary conditions $B(L)=0$. Both $A(z)$ and $B(z)$ are expressed in terms of, and are proportional to, the input beam $A(0)$.

The reflectivity is given by $R=|B(0) / A(0)|^{2}$. It is plotted in Fig. 3 for the case of equal-intensity input beams (during the writing phase) for several values of the grating phase $\phi$. In general, the reflectivity maximum occurs at a different frequency than that of the writing beams; it occurs at a frequency mismatch of $\Delta \beta=g / 2 \cos \phi$; i.e., it occurs at $\Delta \beta^{\prime}=0$. This mismatch arises, again, because the two writing beams influence each other's phase (as well as intensity) by a total amount $\Delta \phi(z)=g \cos (\phi) z$. This increases (or decreases) the spatial frequency of the index grating, altering the frequency at which maximum reflectivity is achieved. The reflectivity plotted in Fig. 3 is symmetric about the line $\Delta \beta=0$ when $\phi$ is reflected

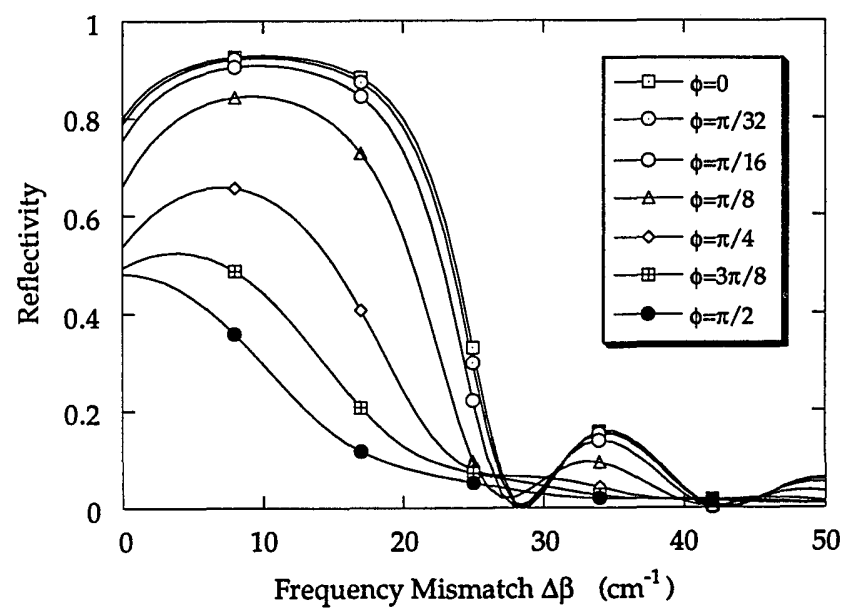

Fig. 3. Reflectivity from the fixed index gratings of Fig. 2. The reflectivity maximum occurs at a frequency mismatch given by $\Delta \beta=g / 2 \cos \phi$. The overall reflectivity, as well as the sidelobes, is reduced by the grating apodization for $\phi>0$. 


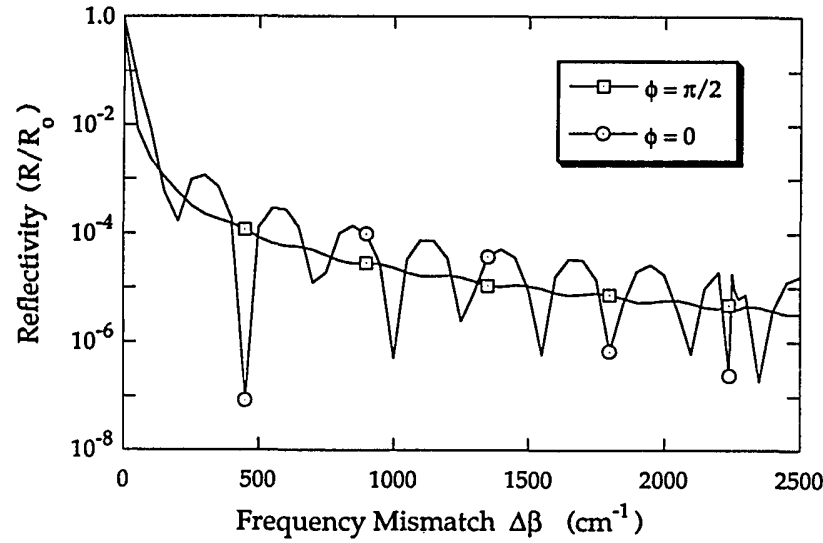

Fig. 4. Reflectivity from the fixed index gratings for $\phi=0$ and $\phi=\pi / 2$ at large values of frequency mismatch. The $\phi=\pi / 2$ grating reflectivity has reduced sidelobes whose peaks are approximately $6 \mathrm{~dB}$ lower than in the $\phi=0$ case (however, the maximum reflectivity is also $3 \mathrm{~dB}$ lower).

about $\pi / 2$. For example, the reflectivity curves for two identical materials, one with $\phi=\pi / 2-x$ and one with $\phi=\pi / 2+x$, are identical except that the position of maximum reflectivity is shifted to a longer wavelength from the writing wavelength in the first case $[\Delta \beta=$ $g \cos (\pi / 2-x) / 2>0]$ and to a shorter wavelength in the second case $[\Delta \beta=g \cos (\pi / 2+x) / 2<0]$. For this reason we have illustrated the reflectivity-versus-frequency mismatch only for $0 \leq \phi \leq \pi / 2$. We note that the grating apodization caused by intensity coupling for $\phi \neq 0$ or $\pi$ leads to slightly reduced sidelobes in the reflectivity and lower overall reflectivity at large values of frequency mismatch. This effect is illustrated in Fig. 4, where the reflectivities of the $\phi=0$ and $\phi=\pi / 2$ cases are compared at large values of mismatch.

\section{Special Case: $\phi=\pi / 2$}

We now consider how the foregoing analysis is simplified under special conditions. For $\phi=\pi / 2$ we note that the beam coupling consists purely of intensity coupling rather than phase coupling. This leads to a simpler form of the index grating [Eq. (11)]; however, little simplification of the final solutions occurs. Since the phase coupling is absent in this case, the reflectivity maximum of the grating occurs at the same frequency as that of the writing beams (see Fig. 3 ).

Special Case: $\phi=0$

The case $\phi=0$ (or $\phi=\pi$ ) is completely different. Here there exists no intensity coupling, whereas the phase coupling is maximum. Hence the magnitude of the index grating is a constant throughout the volume of the crystal. Starting from Eqs. (3), (9), and (10), we obtain, in analogy to Eq. (11),

$$
\Delta n=n_{1}\left[I_{1}(0) I_{2}(0)\right]^{1 / 2} / I e^{i g z} .
$$

The reflectivity in this case is easily shown to be

$$
R=\frac{\kappa^{2} \sinh ^{2} s L}{\kappa^{2} \sinh ^{2} s L+s^{2}},
$$

where

$$
\begin{aligned}
& \kappa=g\left[I_{1}(0) I_{2}(0)\right]^{1 / 2} / I, \\
& s=\left(\kappa^{2}-\delta^{2} / 4\right)^{1 / 2}, \\
& \delta=g-2 \Delta \beta .
\end{aligned}
$$

Equation (29) has the simple form characteristic of Bragg reflection from a constant amplitude grating, ${ }^{6}$ except for the frequency shift in maximum reflectivity, which occurs here at $\Delta \beta=g / 2(\Delta \beta=-g / 2$ when $\phi=\pi)$ rather than at $\Delta \beta=0$. The expression for the reflectivity, Eq. (29), reduces to a familiar result at this point: when $\Delta \beta=g / 2$, we have $R=\tanh ^{2}(\kappa L)$. In Fig. 3, where $\kappa L=2$, we obtain $R=0.92$. Another special case occurs at zero frequency shift: when $\Delta \beta=0$, Eq. (29) reduces to $R=(\kappa L)^{2} /\left[1+(\kappa L)^{2}\right]=0.8$. Thus for this case the phase coupling invokes a penalty of approximately $15 \%$ in the reflectivity when the reading-beam frequency is not shifted.

\section{Special Case: $c=0$}

A case similar to that of $\phi=0$ occurs for arbitrary phase $\phi$ when $c=0$, that is, when the coupled beams are everywhere of equal intensity, although they may vary with $z$. Starting again from Eqs. (4a) and (4b), we derive, in analogy to Eqs. (8) and (10), intensities and phases of the coupled beams,

$$
\begin{aligned}
I_{1}(z) & =I_{2}(z)=v / 2 \exp (-\Gamma z / 2)=I_{1}(0) e(-\Gamma z / 2) \\
\psi_{1}(z) & =-\psi_{2}(z)=\cos (\phi) g z / 2
\end{aligned}
$$

where $v$ is defined in Eq. (9b). From Eqs. (31) and (32) we derive the index grating to be a constant magnitude,

$$
\Delta n(z)=n_{1} / 2 \exp [i g \cos (\phi) z]
$$

In comparing Eq. (33) with Eq. (28), note the important difference between the two cases $c=0$ and $\phi=0$. Here the beams are intensity coupled with a gain coefficient of $g \sin \phi$; this reduces the phase-coupling coefficient from $g$ to $g \cos \phi$. Also note that since here the beams are everywhere of equal intensity, the term $\left[I_{1}(0) I_{2}(0)\right]^{1 / 2} / I$ from Eq. (28) reduces to $1 / 2$ in Eq. (33). Since the formation of the index grating is intensity independent [Eq. (3)], the index grating is constant, although the intensities of the beams are not. In the case $\phi=0$ the beams need not be of equal intensity, because their intensities are constant throughout the volume of the photorefractive medium.

The index grating [Eq. (33)] is fixed in the material. The coupled-mode equations describing reflection become

$$
\begin{aligned}
& A^{\prime}(z)=i \frac{g}{2} e^{i \phi} \exp [i(g \cos \phi-2 \Delta \beta) z] B(z), \\
& B^{\prime}(z)=-i \frac{g}{2} e^{-i \phi} \exp [-i(g \cos \phi-2 \Delta \beta) z] A(z) .
\end{aligned}
$$

These equations are readily solved to yield a reflectivity identical in form to that in the case $\phi=0$ [Eq. (29)], with the modified definitions $\kappa=g / 2$ and $\delta=g \cos \phi-2 \Delta \beta$. 
Special Case: $g_{\text {write }} \neq g_{\text {read }}$

Since the index gratings are assumed to be fixed (possibly by thermal fixing), it is possible that the coupling constants during reading and writing of the holographic grating are not equal $\left(g_{r} \neq g_{w}\right)$. This possibility can be treated with a slight modification of the previous solution. Referring to Eqs. (14) and (16), the dissimilar coupling constants lead to a modification of $\kappa$ to $\kappa^{\prime}=-e^{i \phi} g_{r} /\left(2 g_{w} \sin \phi\right)$; otherwise, Eqs. (14) are unaltered. The solutions obtained in this case are identical to those of Eqs. (25), with the change $\beta \rightarrow \beta^{\prime}=\left[g_{r}{ }^{2} /\left(4 g_{w}{ }^{2} \sin ^{2} \phi\right)-\eta^{2}\right]^{1 / 2} / 2$. The coefficients in Eqs. (26) and (27) are also altered. Coefficients $C_{1}$ and $D$ each contain a term $4 \sin ^{2} \phi$, which must be replaced by $\left(4 g_{w}{ }^{2} \sin ^{2} \phi\right) / g_{r}{ }^{2}$, and coefficients $C_{3}$ and $C_{4}$ each must be multiplied by $g_{w} / g_{r}$. With these changes the original solution holds.

\section{SPECTRAL RESPONSE, LOSSY CASE}

When the loss in the material is considered, the equations become substantially more complicated. Analytic expressions are obtained only for dynamic holography and only for $\phi=0$. Reflectivities of the fixed holograms are calculated numerically. We start with the dynamic coupled-mode equations, as in Eqs. (4a) and (4b):

$$
\begin{aligned}
& A^{\prime}(z)=i g|B(z)|^{2} / I(z) e^{i \phi} A(z)-(\alpha / 2) A(z), \\
& B^{\prime}(z)=-i g|A(z)|^{2} / I(z) e^{-i \phi} B(z)+(\alpha / 2) B(z)
\end{aligned}
$$

Performing the same transformation as in Eqs. (5), we are led to the following equations for the phases and magnitudes of the coupled beams:

$$
\begin{aligned}
a^{\prime}(z) & =-\sin (\phi) g|b(z)|^{2} / I(z)[a(z)]-(\alpha / 2) a(z), \\
b^{\prime}(z) & =-\sin (\phi) g|a(z)|^{2} / I(z)[b(z)]+(\alpha / 2) b(z), \\
\psi_{1}^{\prime}(z) & =\cos (\phi) g|b(z)|^{2} / I(z), \\
\psi_{2}^{\prime}(z) & =-\cos (\phi) g|a(z)|^{2} / I(z)
\end{aligned}
$$

Again, the special case $\phi=0$ yields considerably simplified formulas. In this special case, analytic solutions of Eqs. (36) and (37) are obtained easily. They are

$A(z)=A(0) \exp (-\alpha z / 2)\left[\frac{B(0)^{2} \exp (2 \alpha z)+A(0)^{2}}{B(0)^{2}+A(0)^{2}}\right]^{(i g) /(2 \alpha)}$

$$
\begin{aligned}
B(z)= & B(0) \exp (+\alpha z / 2) \\
& \times\left[\frac{B(0)^{2}+A(0)^{2} \exp (-2 \alpha z)}{B(0)^{2}+A(0)^{2}}\right]^{(i g) /(2 \alpha)} .
\end{aligned}
$$

Thus the index grating is given by

$$
\Delta n(z)=n_{1} \frac{A(0) B(0) \exp (i g z)}{A(0)^{2} \exp (-\alpha z)+B(0)^{2} \exp (\alpha z)} .
$$

When this grating is fixed, we can formulate the coupled equations describing reflectivity in analogy to Eqs. (12), using Eqs. (35). One can manipulate these equations by following steps identical to those in the analysis up to Eqs. (19). In this case, however, instead of the Pöschl-Teller equation [Eq. (A9) below], the secondorder equation obtained is a symmetric top equation. ${ }^{15,16}$ We do not present its solution here, since it is useful only for $\phi=0$.

Alternatively, we solved Eqs. (35) numerically for arbitrary photorefractive phase $\phi$ in order to obtain the index grating that is fixed in the material. This calculated index grating is then fitted to a high-order polynomial (usually twelfth order), and that polynomial approximation is then used to calculate the reflectivity numerically from the reflectivity equations. In solving the dynamic equations, we specify boundary conditions at $z=0$; i.e., $A(0)$ and $B(0)$. The $B(0)$ is iteratively modified to yield the desired value for $B(L)$, which is the physical input to the crystal. When the reflectivity was calculated, we used the standard procedure of fixing $B(L)=0$ and $A(L)=$ 1 and working backward toward $z=0 .{ }^{11}$ Finally, the reflectivity is normalized relative to the numerically computed $A(0)$ [where $|A(0)|>1$ ]. The addition of a nonzero loss has two dramatic effects on the results: first, the reflectivity of the filter is now strongly nonreciprocal; second, since energy is no longer conserved, the quantity $|A(z)|^{2}-|B(z)|^{2}$ is no longer constant. In fact, the intensities can become equal at any arbitrary point within the

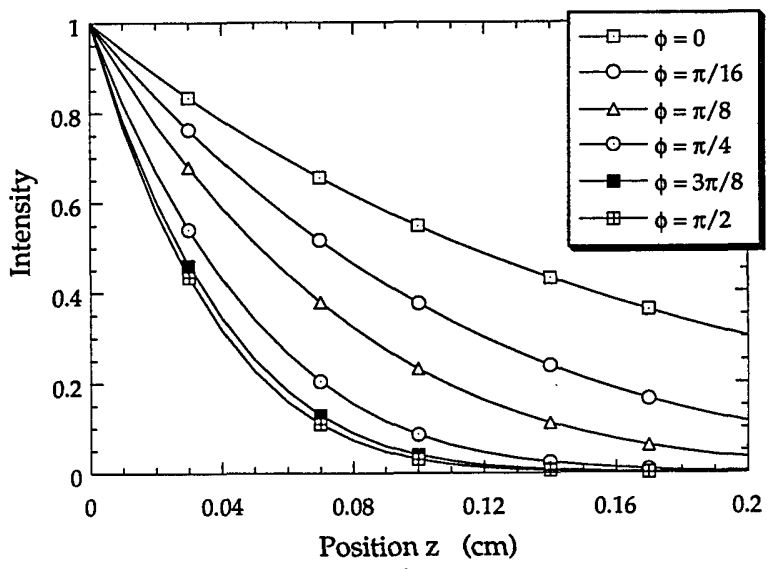

(a)



(b)

Fig. 5. (a) Intensity of beam 1 incident at $z=0$ for various values of $\phi$, for a loss constant of $\alpha=6 / \mathrm{cm}$, and for equal-intensity inputs. The coupling constant is $g=20 / \mathrm{cm}$, and the crystal length is $L=0.2 \mathrm{~cm}$. (b) Intensity of beam 2 incident at $z=L$ under conditions identical to those for (a). 


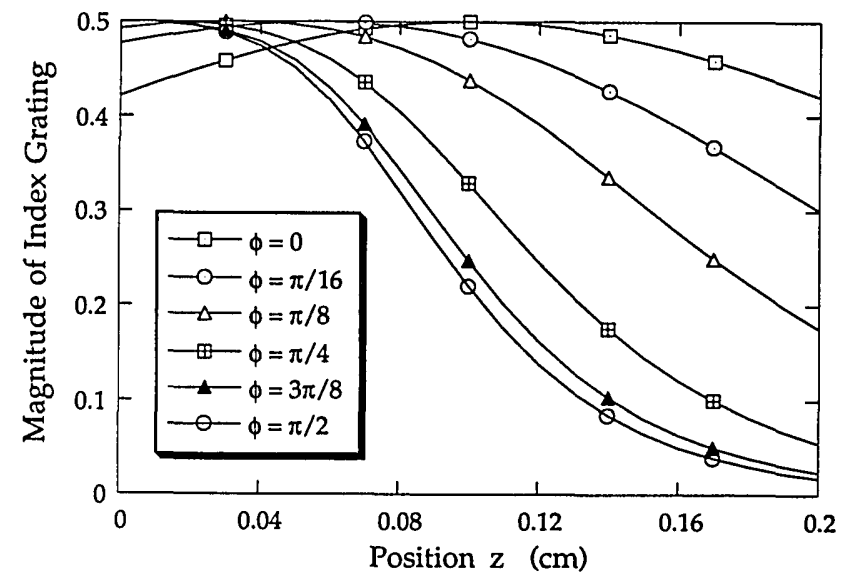

Fig. 6. Index grating formed in the crystal by the coupled beams of Fig. 5. The effect of the loss is to eliminate the no-crossing rule for the two beam intensities. Thus when $g / 2 \sin \phi \leq \sim \alpha$, the intensities can be equal within the volume of the crystal. This equality leads to a maximum in the magnitude of the index grating located away from the edge of the crystal.

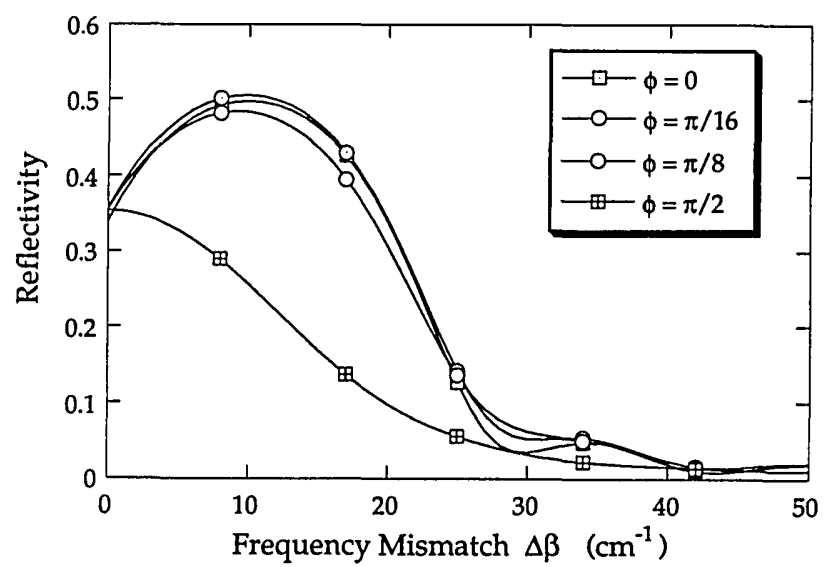

Fig. 7. Reflectivity for the case $\alpha=6 / \mathrm{cm}$ versus frequency mismatch for a beam incident at $z=0$ upon the index gratings of Fig. 6. The behavior is complex because the magnitude of the index grating near $z=0$ (the most efficient region of reflection) first increases and then decreases with increasing photorefractive grating phase $\phi$.

volume of the crystal. Thus the index grating may have its maximum at any point in the volume of the crystal rather than only at the entrance or exit facet.

In the following calculations we use, as above, a coupling constant $g=20 / \mathrm{cm}$, and a crystal length of $L=$ $0.2 \mathrm{~cm}$, and we assume equal-intensity inputs $A(0)$ and $B(L)$. The loss coefficient is taken to be $\alpha=6 / \mathrm{cm}$. First the equations for the dynamically coupled beams $A(z)$ and $B(z)$ are computed from Eqs. (35a) and (35b), respectively. The results for various values of the grating phase $\phi$ are shown in Fig. 5. The nonzero loss affects mainly the shape of $B(z)$, incident from $z=L$. This occurs because the beam-coupling and loss mechanisms are opposed. Thus $B(z)$ has a minimum at the position at which the material loss is balanced by the beam coupling. Also, the loss allows the two beams to have equal intensities at one point in the volume of the crystal. From Eqs. (36) we determine that this condition is permitted approximately when $g / 2 \sin \phi<\alpha$. The index grating formed by the two beams (Fig. 6) illustrates this effect. For $\phi=0$ it is clear that the index grating is maximum in the center of the crystal.

Next we calculate numerically the reflectivity from the index gratings of Fig. 6 . Figure 7 shows the reflectivity for a beam incident upon the $z=0$ side of the crystal, and Fig. 8 shows the reflectivity under the same conditions for a beam incident at $z=L$. Since the index grating is, in general, stronger near $z=0$, the reflectivity is higher for a beam incident at $z=0$. The difference between the two cases is most pronounced when the grating phase $\phi$ is near $\pi / 2$. Also, we point out that the behavior of the reflectivity as $\phi$ increases is more complex in the case of reflection from the $z=0$ side. This occurs because the index grating near $z=0$ (the most efficient region of reflection for a beam incident at $z=0$ ) first increases and then decreases with increasing photorefractive phase $\phi$. We can see how this effect arises, by an inspection of the shape of $B(z)$ in Fig. 5(b).

\section{COMPARISON OF THEORY WITH EXPERIMENT}

We compare the theory of spectral response of photorefractive gratings obtained in the preceding sections with recently reported results of a solar $\mathrm{H}-\alpha$ photorefractive interference filter marketed by Accuwave, Inc. ${ }^{17}$ Lithium niobate was used as the photorefractive material; its thickness is $1.2 \mathrm{~cm}$. The center wavelength of transmission is $656.46 \mathrm{~nm}$ with a peak reflectivity taken equal to $R=55 \%$ and a $3-\mathrm{dB}$ bandpass (full width at halfmaximum) of $0.125 \mathrm{~nm}$. In Fig. 9 two theoretical filter responses are superimposed upon the experimentally determined response of the Accuwave filter. We chose the writing-beam wavelengths, the coupling constant $g$, the photorefractive phase $\phi$, and the optical absorption $\alpha$ to match the peak reflectivity wavelength and magnitude, as well as the full width at half-maximum. Rough values for the experimental parameters were provided by George Rakuljic of Accuwave Corporation in a private communication. ${ }^{18}$ For the upper curve we used $g=$ $3 / \mathrm{cm}, \phi=\pi / 3$, and for the lower curve we used $g=$

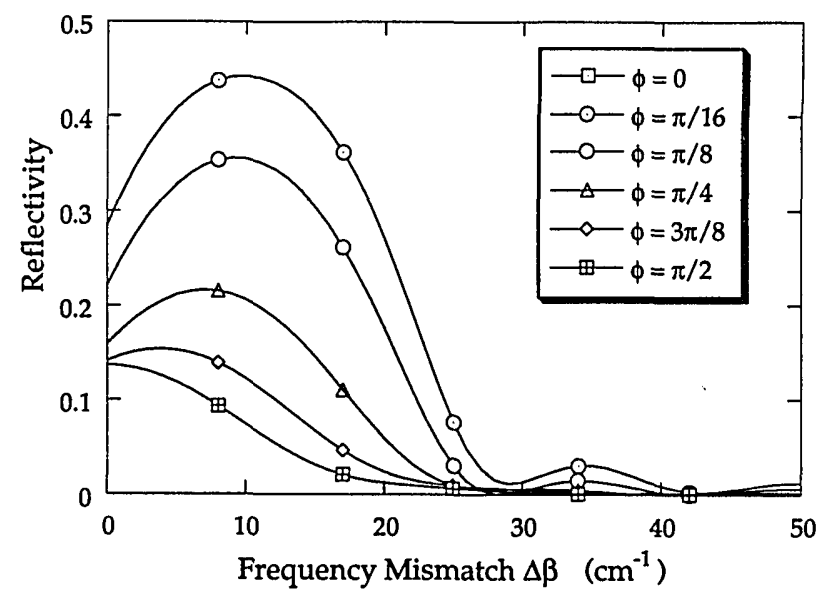

Fig. 8. Reflectivity for the case $\alpha=6 / \mathrm{cm}$ versus frequency mismatch for a beam incident at $z=L$ upon the index gratings of Fig. 6. Note the strong nonreciprocity in comparison with Fig. 7. The behavior for this case is simpler than that of Fig. 7, because the magnitude of the index grating near $z=L$ (the most efficient region of reflection) decreases monotonically with increasing photorefractive grating phase $\phi$. 


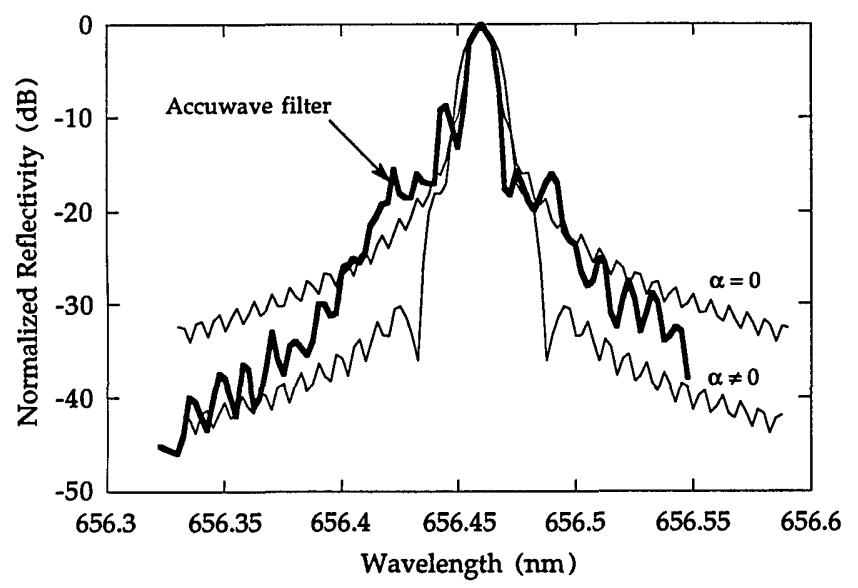

Fig. 9. Reflectivity of the Accuwave $\mathrm{H}-\alpha$ filter (heavy curve) compared with theoretical plots (light curves). For the upper curve, $g=3 / \mathrm{cm}, \phi=\pi / 3$, and $\alpha=0$; for the lower curve, $\mathrm{g}=5 / \mathrm{cm}, \phi=\pi / 6, \alpha_{\text {write }}=4 / \mathrm{cm}$, and $\alpha_{\text {read }}=0.5 / \mathrm{cm}$.

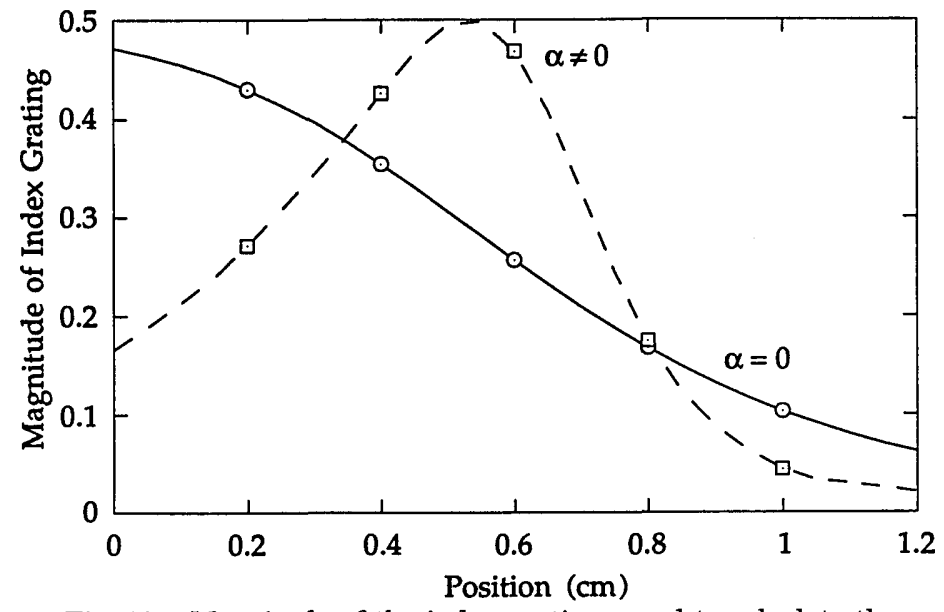

Fig. 10. Magnitude of the index gratings used to calculate the reflectivities of Fig. 9. The index grating in the case of nonzero optical absorption is more strongly apodized.

$5 / \mathrm{cm}, \phi=\pi / 6, \alpha_{\text {read }}=0.5 / \mathrm{cm}$, and $\alpha_{\text {write }}=4 / \mathrm{cm}$. The reflectivity is calculated for beams incident at $z=L$ (the weak reflection). Both curves match the Accuwave filter well near $\Delta \beta=0$; however, only the curve $\alpha \neq 0$ fits well for large values of the frequency mismatch.

The deviation of the upper curve from the experimental data is significant because the decay of the reflectivity sidelobes at large values of frequency mismatch determines the performance of the filter. If the hologram were used instead to store a page of data, the sidelobes would determine how closely adjacent pages of data could be written and thus the information density of the material. We conclude that the Accuwave filter performs substantially better than a lossless theoretical filter.

The second curve is generated with the inclusion of optical absorption. The values of $\alpha$ during writing and reading were chosen on the basis of discussions with Accuwave representatives on the fabrication process of the filter. Although this curve behaves similarly for small $\Delta \beta$, it has dramatically reduced sidelobes. As mentioned above, it is possible for $g_{\text {read }}$ and $g_{\text {write }}$ to be different, also; however, this complication was determined not to affect the output substantially.
The improved performance of the lower curve is quite easy to understand by reference to Figs. 6 and 2 for the index grating in a photorefractive material with and without optical absorption, respectively, and also to Fig. 10, which illustrates the index gratings used to generate Fig. 9. As described above, when optical absorption is ignored, the maximum amplitude of the index grating always occurs at the entrance facet. When the loss is included, it is possible to get the maximum of the index grating within the volume of the crystal. In the dashed curve of Fig. 10, the maximum is very near the center of the crystal. Thus the index grating is truncated more gently (smaller discontinuity) at the edges of the crystal when optical absorption is present. The large discontinuity in the index grating at the edges is responsible for the large reflectivity sidelobes when $\alpha=0$.

We can understand this by an inspection of Eq. (1) or (34) in the limit of large $\Delta \beta$. Here $A(z)$ is approximately constant and the reflectivity is weak. It is clear that the output $B(0)$ is given approximately by the Fourier transform of $\Delta n(z)$. Thus, as in Fig. 4, if one index grating is of uniform amplitude and the other is smoothly truncated on one facet and discontinuous on the other, we expect a 3-dB difference between the sidelobe amplitudes of the gratings. In the case of Figs. 9 and 10, when absorption is included, $B(0)$ becomes the Fourier transform of $\Delta n \exp (-2 \alpha z)$ or $\Delta n \exp [-2 \alpha(L-z)]$ for beams incident at $z=0$ or $z=L$, respectively. Thus the reflectivity from the $z=L$ side of the strongly apodized index grating should have much weaker sidelobes than the zero-loss index grating. This is exactly the behavior that we observed, and we conclude that the optical absorption is a key ingredient in the superior performance of the Accuwave filter.

\section{SUMMARY}

In summary, we describe an analytic solution for the frequency response of interference filters written with the photorefractive effect. We allow for an arbitrary photorefractive grating phase $\phi$. In addition we present numerical solutions of the identical procedure with a nonzero optical absorption in the material. A number of aspects of the interplay between the intensity and the phase coupling that are due to $\phi$ are discussed. Some of the results are applied to experimental data of fixed holographic gratings in $\mathrm{LiNbO}_{3}$ for which it is determined that the optical absorption of the medium improves the filter response.

\section{APPENDIX A: TRANSFORMATION OF COUPLED DIFFERENTIAL EQUATIONS}

In the study of beam coupling and holography in photorefractive materials, one often encounters equations of the form

$$
\begin{aligned}
& A^{\prime}(z)=i \kappa f_{1}(z) \exp (i \delta z) B(z), \\
& B^{\prime}(z)= \pm i \kappa^{*} f_{2}(z) \exp (-i \delta z) A(z) .
\end{aligned}
$$

Here we define both $f_{1}(z)$ and $f_{2}(z)$ as possessing the same sign, usually $f_{i}(z)>0$. For beam-coupling problems, the plus occurs when the copropagating (transmission) ge- 
ometry is used; the minus occurs in the counterpropagating (reflection) geometry. The typical method of solving this type of equation set is to differentiate either equation, obtaining a second-order equation, and then substitute the remaining equation into the second-order equation to obtain a second-order equation that contains terms of only one dependent variable, i.e., $A(z)$ and $A^{\prime \prime}(z)$ or $B(z)$ and $B^{\prime \prime}(z)$, but no first-derivative terms.

The presence of the $\delta$ term and the $f_{1}(z)$ and $f_{2}(z)$ terms in Eq. (A1) complicate the use of this method, since they contribute terms to the derivative. In general, after following the procedure outlined above, we are left with second-order equations containing terms $A(z), A^{\prime}(z)$, and $A^{\prime \prime}(z)$. The difficulty in solving the equations is that the coefficients of these terms are functions of $z$. In order to overcome this problem we seek a simplifying transformation of the dependent variables of Eqs. (A1) that will permit the equations to be solved.

First we seek a transformation $A(z), B(z) \rightarrow T(z)$, $V(z)$, which permits the expression of Eqs. (A1) to be expressed as two second-order equations without $T^{\prime}(z)$ or $V^{\prime}(z)$ terms. We perform the transformation

$$
\begin{aligned}
& A(z)=T(z) \exp [i F(z)], \\
& B(z)=V(z) \exp [-i F(z)],
\end{aligned}
$$

(A2b)

where $F(z)$ is a function to be determined. Equations (A1a) and (A1b) become, respectively (with the minus),

$$
\begin{aligned}
& T^{\prime}(z)=i \kappa V(z) \exp \{i[\delta z-2 F(z)]\} f_{1}(z)-i F^{\prime}(z) T(z) \\
& V^{\prime}(z)=-i \kappa^{*} T(z) \exp \{i[2 F(z)-\delta z]\} f_{2}(z)+i F^{\prime}(z) V(z)
\end{aligned}
$$

When Eq. (A3a) is differentiated and Eq. (A3b) is used to eliminate terms containing $V(z)$ and $V^{\prime}(z)$, we obtain

$$
\begin{aligned}
T^{\prime \prime}(z)= & {\left[i \delta-2 i F^{\prime}(z)+f_{1}^{\prime}(z) / f_{1}(z)\right] T^{\prime}(z) } \\
& +\left\{|\kappa|^{2} f_{1}(z) f_{2}(z)-\delta F^{\prime}(z)+\left[F^{\prime}(z)\right]^{2}\right. \\
& \left.+i F^{\prime}(z) f_{1}^{\prime}(z) / f_{1}(z)-i F^{\prime \prime}(z)\right\} T(z)
\end{aligned}
$$

The $T^{\prime}(z)$ term vanishes when $F^{\prime}(z)=\delta / 2+$ $f_{1}^{\prime}(z) /\left[2 i F_{1}(z)\right]$, or

$$
F(z)=\frac{\delta z}{2}+\frac{\ln \left[f_{1}(z)\right]}{2 i}
$$

An analysis of $V^{\prime \prime}(z)$ similar to that leading up to Eq. (A4) gives the condition

$$
F(z)=\frac{\delta z}{2}-\frac{\ln \left[f_{2}(z)\right]}{2 i}
$$

in order that the $V^{\prime}(z)$ term be zero. Comparison of Eqs. (A5) and (A6) shows that the two conditions can be met simultaneously if and only if

$$
f_{2}(z)=1 / f_{1}(z)
$$

When $f_{1}(z)$ is on the unit circle [as in Eq. (15)], the stipulation of Eq. (A7) reduces to $f_{2}(z)=f_{1}{ }^{*}(z)$. When condition (A7) is satisfied, Eqs. (A3a) and (A3b) can be written as

$$
\begin{aligned}
& T^{\prime \prime}(z)=\left\{|\kappa|^{2}-\left[F^{\prime}(z)\right]^{2}-i F^{\prime \prime}(z)\right\} T(z), \\
& V^{\prime \prime}(z)=\left\{|\kappa|^{2}-\left[F^{\prime}(z)\right]^{2}+i F^{\prime \prime}(z)\right\} V(z),
\end{aligned}
$$

respectively. If we had taken the plus in Eqs. (A3), then Eqs. (A8) would be modified by replacement of $|\kappa|^{2}$ with $-|\kappa|^{2}$. The transformation described above will be referred to as the corotating transform.

The advantages of expressing the coupled equations in this way are that many second-order equations of the form of Eqs. (A8) have been solved and their solutions can be referenced. ${ }^{16}$ In the text it is shown that when the coupled equations describing photorefractive beam coupling are subjected to the procedure described above, the two second-order equations that result are of the form

$$
\psi^{\prime \prime}(r)=\alpha^{2}\left[\frac{\kappa(\kappa-1)}{\sinh ^{2}(\alpha r)}-\frac{\lambda(\lambda+1)}{\cosh ^{2}(\alpha r)}+(21-1)^{2}\right] \psi(r) .
$$

This form is the second Pöschl-Teller equation, which has been solved ${ }^{14}$ with a modification of the factorization method. ${ }^{15}$ The equation is used to describe the bound and scattering states of diatomic molecules, hence the quantum-mechanical notation.

The method of solution will not be described here (see Ref. 14); instead, we merely list the solutions. Two eigenfunctions are solutions of Eq. (A9):

$$
\begin{aligned}
\psi_{1}(r)= & \sinh ^{1-\kappa}(\alpha r) \cosh ^{\lambda+1}(\alpha r)_{2} F_{1}\left[\left(\frac{\lambda-\kappa}{2}-l+1\right)\right. \\
& \left.+\frac{1}{2},\left(\frac{\lambda-\kappa}{2}+l\right)+\frac{1}{2} ; \frac{3}{2}-\kappa ;-\sinh ^{2}(\alpha r)\right],
\end{aligned}
$$

$$
\begin{aligned}
\psi_{2}(r)= & \sinh ^{\kappa}(\alpha r) \cosh ^{\lambda+1}(\alpha r) \\
& \times{ }_{2} F_{1}\left[\left(\frac{\lambda+\kappa}{2}-l+1\right),\left(\frac{\lambda+\kappa}{2}+l\right) ; \frac{1}{2}+\kappa ;\right. \\
& \left.-\sinh ^{2}(\alpha r)\right],
\end{aligned}
$$

where ${ }_{2} F_{1}[\ldots]$ is the hypergeometric function, defined by

$$
\begin{aligned}
{ }_{2} F_{1}[a, b ; c ; z] & ={ }_{2} F_{1}[b, a ; c ; z] \\
& =\frac{\Gamma(c)}{\Gamma(a) \Gamma(b)} \sum_{n=0}^{\infty} \frac{\Gamma(a+n) \Gamma(b+n)}{\Gamma(c+n)} \frac{z^{n}}{n !} .
\end{aligned}
$$

The particular solutions of first-order coupled equations (A1) that led to the second-order Pöschl-Teller equations will consist of linear combinations of the eigenfunctions $\psi_{1}$ and $\psi_{2}$, with coefficients determined by boundary conditions. Calculation of the coefficients is generally tedious. Use is made of the Gauss transformations for hypergeometric functions, ${ }^{19}$ the differential relation

$$
\frac{\mathrm{d}}{\mathrm{d} z}{ }_{2} F_{1}[a, b ; c ; z]=\frac{a b}{c}{ }_{2} F_{1}[a+1, b+1 ; c+1 ; z],
$$

and the linear transformation

$$
{ }_{2} F_{1}[a, b ; c ; z]=(1-z)^{c-a-b}{ }_{2} F_{1}[c-a, c-b ; c ; z] .
$$




\section{ACKNOWLEDGMENTS}

The authors thank the U.S. Army Research Office (Durham, N.C.), the U.S. Air Force Office of Scientific Research, and the Advanced Research Projects Agency for supporting this work.

*Permanent address, NTT Interdisciplinary Research Laboratories, Ibaraki, Japan.

\section{REFERENCES}

1. G. Rakuljic, A. Yariv, and V. Leyva, "High resolution volume holography using orthogonal data storage," in Photorefractive Materials, Effects, and Devices, Vol. 14 of 1991 OSA Technical Digest Series (Optical Society of America, Washington, D.C., 1991), paper MD-3.

2. G. Rakuljic, V. Leyva, and A. Yariv, "Optical data storage using orthogonal wavelength multiplexed volume holograms," Opt. Lett. 17, 1471-1473 (1992).

3. V. Leyva, G. Rakuljic, and A. Yariv, "Volume holography using orthogonal data storage approach," in OSA Annual Meeting, Vol. 17 of 1991 OSA Technical Digest Series (Optical Society of America, Washington, D.C., 1991), paper FU-7.

4. G. Rakuljic, V. Leyva, and A. Yariv, "Comparison of angle and wavelength multiplexing in holographic data storage (invited paper)," in OSA Annual Meeting, Vol. 23 of 1992 OSA Technical Digest Series (Optical Society of America, Washington, D.C., 1992), paper WE-2.

5. J. O. White, S. Z. Kwong, M. Cronin-Golomb, B. Fischer, and A. Yariv, "Wave propagation in photorefractive media," in Photorefractive Materials and Their Applications II, P. Günther and J. P. Huignard, eds. (Springer-Verlag, Berlin, 1989), Chap. 4.
6. A. Yariv, Optical Electronics, 4th ed. (Saunders, Philadelphia, 1991), p. 496.

7. T. J. Hall, R. Jaura, L. M. Connors, and P. D. Foote, "The photorefractive effect: a review," Prog. Quantum Electron. 10, 77-146 (1985).

8. P. Yeh, "Contra-directional two-wave mixing in photorefractive media," Opt. Commun. 45, 323-326 (1983).

9. Y. H. Ja, "Energy transfer between two beams in writing a reflection volume hologram in a dynamic medium," Opt. Quantum Electron. 14, 547-556 (1982).

10. Ref. 6, p. 494.

11. C. Gu and P. Yeh, "Diffraction properties of fixed gratings in photorefractive media," J. Opt. Soc. Am. B 7, 2339-2346 (1990).

12. M. Segev, A. Kewitsch, A. Yariv, and G. Rakuljic, "Selfenhanced diffraction from fixed photorefractive gratings during coherent reconstruction," Appl. Phys. Lett. 62, 907-909 (1993).

13. J. H. Hong and R. Saxena, "Diffraction efficiency of volume holograms written by coupled beams," Opt. Lett. 16, 180-182 (1991).

14. A. O. Barut, A. Inomata, and R. Wilson, "Algebraic treatment of second Pöschl-Teller, Morse-Rosen, and Eckart equations," J. Phys. A 20, $4083-4090$ (1987).

15. L. Infeld and T. E. Hull, "The factorization method," Rev. Mod. Phys. 23, 21-68 (1951).

16. D. Zwillinger, Handbook of Differential Equations (Academic, New York, 1989).

17. G. A. Rakuljic and V. Leyva, "Volume holographic narrowband optical filter," Opt. Lett. 18, 459-461 (1993).

18. G. Rakuljic, 1653 Nineteenth Street, Santa Monica, Calif. 90404 (personal communication, May 1993).

19. A. Abramowitz and I. Stegun, Handbook of Mathematical Functions (Dover, New York, 1972), Chap. 15. 